\title{
Specific Learning Disorder: Challenges and Intricacies for a Practicing Psychiatrist
}

\author{
${ }^{1}$ Vimal Doshi Veerappan
}

How to Cite this Article: Vimal Doshi V. Specific Learning Disorder: Challenges and Intricacies for a Practicing Psychiatrist. Indian Journal of Mental Health and Neurosciences. 2021;4(3): pp 1-2

0 pecific Learning Disorder (SLD) is one of the common diagnoses often encountered in psychiatry $^{(1)}$. As with many neurodevelopmental disorders early identification, specific intense remediation, appropriate management of comorbid conditions, coordination with teachers and parents and engaging the child long-term are the mainstay of management of SLD. Each of these steps pose unique challenges due to the inherent complexity of SLD, lack of uniform, standardised curriculum and testing methods, wide regional variations in definition, assessment and certification in SLD, strained relationship between professionals of various disciplines and lack of custommade tools for screening, assessment and follow-up in native (Indian) languages ${ }^{(2)}$.

The initial diagnosis of SLD begins with a psychiatrist exploring various other causes for poor academic performance. A practicing clinician should always be vigilant about various neuropsychiatric conditions and/or the medications prescribed for various medical conditions that can contribute to cognitive deficit leading to recent (less than 3 months) or sudden academic decline. Other neurodevelopmental disorders (such as ADHD) or a psychiatric illness (such as OCD) can directly or indirectly worsen the academic ability thereby posing an additional layer to be dealt with before unmasking the true learning (dis)ability of the child. A common overlooked aspect is the educational factors including medium, quality, structure of pedagogy, school attendance, parental involvement and standardised assessment methods which can contribute to the level of academic achievement. Finally, SLD cannot be diagnosed in the presence of Intellectual Disability or Sensory impairments (visual and auditory) ${ }^{(3)}$.

Recurrent abdominal pain, unexplained headaches, repeated hyperventilation episodes are some of the common presenting symptoms of Learning Disorders in children who cannot express their academic difficulty. Irritability, truancy, school refusal, oppositional behaviour, conduct symptoms are all associated with academic decline and can be a presenting symptom of SLD in some children. ADHD and anxiety disorders are the most common comorbidities. Parental stress and anxiety and its effect on child's mental status should also be taken into account during evaluation.

Coordination and cooperation between professionals of various disciplines notably, educational psychologists, remedial educators, language pathologists and occupational therapists is essential in identifying the specific deficit(s) such as auditory processing or fine motor in-coordination contributing to the language processing (dis)ability. Psychiatrist should function here as a facilitator with the common goal being increasing the academic ability of the child in the least restrictive method as possible. Making sense of various assessment reports and identifying the core deficit(s) leading to appropriate individualized educational plan and therapy for the child is a challenge by itself without bringing in the factor of availability and affordability of those services to the child and its family ${ }^{(4)}$.

Local educational curriculum, availability and distribution of assessment and therapeutic services, awareness and knowledge of teachers and parents about SLD, Union and State laws pertaining to SLD diagnosis,

${ }^{1}$ Senior Assistant Professor, Institute of Mental Health, Madras Medical College, Kilpauk, Chennai - 600010, Tamilnadu, India. 
categorization and certification and the operational definitions, guidelines and implementation of those guidelines and their amendments play a crucial role in diagnosing and managing SLD. Psychiatrists are in a pivotal position to recommend the best possible treatment practices which are feasible taking into account the ground realities and practices not only for the individual child but also for the future shaping of policies.

Choosing an alternative curriculum for a child with severe math difficulty not responding to intense one to one remedial instruction after sufficient duration is the logical next step, but in practice balancing the emotions of an expected parent, availability of an alternative but equivalent syllabus and empathetic understanding and explanation to the child regarding the change of syllabus and its career options thereby gaining the acceptance and support of the child and the family is an art by itself ${ }^{(5)}$. Delivering a news that a particular child has a dis-ability (say I.Q of 65) but the disability is not eligible for the required educational concession to a concerned parent and managing the emerging emotions but at the same time suggesting alternate best course of action for the child's future is an unenviable task faced frequently when dealing poor academic performance.
Lack of a standardised screening instrument that can be administered by an elementary school teacher for early identification is only one of the lacunae in the assessment of SLD. Others include lack of local language screening instruments, lack of standardised testing for various grades, lack of clear-cut guidelines for categorising into mild moderate and severe forms of SLD, scarcity of inclusive schools and the required specialists in those schools. Thus, often a psychiatrist is left with inaccurate, imprecise instruments and an onus is placed on clinical evaluation, based on which the entire diagnosis is made. This clearly indicates that more research taking into account linguistic, cultural and educational factors and developing instruments based on these needs is an immediate and imperative step.

SLD with its multitude of presentations, many differential diagnoses, difficulties in assessments, lack of appropriate guidelines with scarcity of qualified professionals poses a unique challenge for the practicing psychiatrist. Psychiatrists should wear the coat of a medical specialist, coordinator (between various professionals), research scholar and policy maker without compromising on the goal of the treatment: viz., the child's welfare in a least restrictive path..

\section{REFERENCES}

1 Moll K, Kunze S, Neuhoff N, et al. Specific learning disorder: Prevalence and gender differences. PLoSOne. 2014;9(7):e103537

2 Shaywitz SE, ModyM, \& Shaywitz BA. Neural mechanisms in dyslexia. Current Directions in Psychological Science, 2006;15(6):278-281

3 American Psychiatric Association. (2013). Diagnostic and statistical manual of mental disorders (5th ed.). Washington, DC

4 National Institutes of Health. (2014a). What are learning disabilities? Retrieved from [https://www.nichd.nih.gov/health/topics/learning/conditioninfo/Pages/default.aspx]

5 Ashkenazi S, BlackJM, Abrams DA, et al. Neurobiological underpinnings of math and reading learning disabilities. Journal of Learning Disabilities. 2013;46(6):549-569 